\title{
AstraLux: the Calar Alto Lucky Imaging Camera
}

\author{
Felix Hormuth, Stefan Hippler, Wolfgang Brandner, Karl Wagner, Thomas Henning \\ Max-Planck-Institut für Astronomie, Königstuhl 17, 69117 Heidelberg, Germany
}

\begin{abstract}
AstraLux is the Lucky Imaging camera for the Calar Alto 2.2-m telescope, based on an electron-multiplying high speed CCD. By selecting only the best 1-10\% of several thousand short exposure frames, AstraLux provides nearly diffraction limited imaging capabilities in the SDSS $i$ ' and $z^{\prime}$ filters over a field of view of $24 \times 24$ arcseconds. By choosing commercially available components wherever possible, the instrument could be built in short time and at comparably low cost. We present the instrument design, the data reduction pipeline, and summarise the performance and characteristic: $*$.
\end{abstract}

Keywords: Lucky Imaging, EMCCD, low light level CCD, high angular resolution techniques, diffraction limited imaging, high speed photometry

\section{INTRODUCTION}

The recovery of the full theoretical resolution of large ground-based optical telescopes in the presence of atmospheric turbulence has been a major goal of technological developments in the field of astronomical instrumentation in the past decades. The de-facto standard nowadays is adaptive optics, the active real-time correction of the incoming distorted wavefronts by means of deformable mirrors. While diffraction limited image quality can now be routinely achieved in the near infrared at large telescopes, this is only possible with considerable technical effort.

Even before the age of adaptive optics, speckle interferometric techniques gave access to the diffraction limit of large telescopes by applying auto- or cross-correlation methods in Fourier space to large sets of atmospherically degraded short-time exposures. ${ }^{1-5}$

An even more simple approach has been employed especially by amateur astronomers in order to produce sharp images of the moon or bright planets: instead of using all images of a large set of short-time exposures, only those showing little image distortion due to the variable strength of atmospheric turbulence are combined to a high-resolution result. Like in the speckle interferometric approach, this requires only a detector with fast readout capability and moderate computational effort. The application of this technique to fainter astronomical targets, e.g. low-mass double stars, was limited by the readout noise of the available detectors at visible wavelengths until a few years ago. At typical exposure times of few ten milliseconds - necessary to "freeze" the effects of atmospheric turbulence - photon noise limited detectors are obligatory to use this so-called "Lucky Imaging" technique on targets fainter than $\approx 10 \mathrm{mag}$. Since the image quality of each single frame has to be determined, e.g. by measuring the Strehl ratio of a reference star, the readout noise of the detector sets stringent limits on the minimum brightness of this reference.

The advent of electron multiplying CCDs (EMCCD $)^{6-8}$ led to considerable interest in the Lucky Imaging technique on the part of professional astronomers. First experiments at the Nordic Optical Telescope (NOT) with LuckyCam $^{9-12}$ proved that Lucky Imaging is a very promising alternative to adaptive optics, allowing diffraction limited imaging at telescopes in the $2-3 \mathrm{~m}$ class at wavelengths below $1 \mu \mathrm{m}$. These results triggered the development of a similar instrument for the 2.2-m telescope at Calar Alto by our group. The Lucky Imaging camera AstraLux was built in less than 5 months thanks to the availability of most parts as off-the-shelf equipment.

Further author information: (Send correspondence to F. Hormuth)

F. Hormuth: E-mail: hormuth@mpia.de, Telephone: +49 (0)6221 528221

*Based on observations collected at the Centro Astronómico Hispano Alemán (CAHA) at Calar Alto, operated jointly by the Max-Planck Institut für Astronomie and the Instituto de Astrofísica de Andalucía (CSIC) 

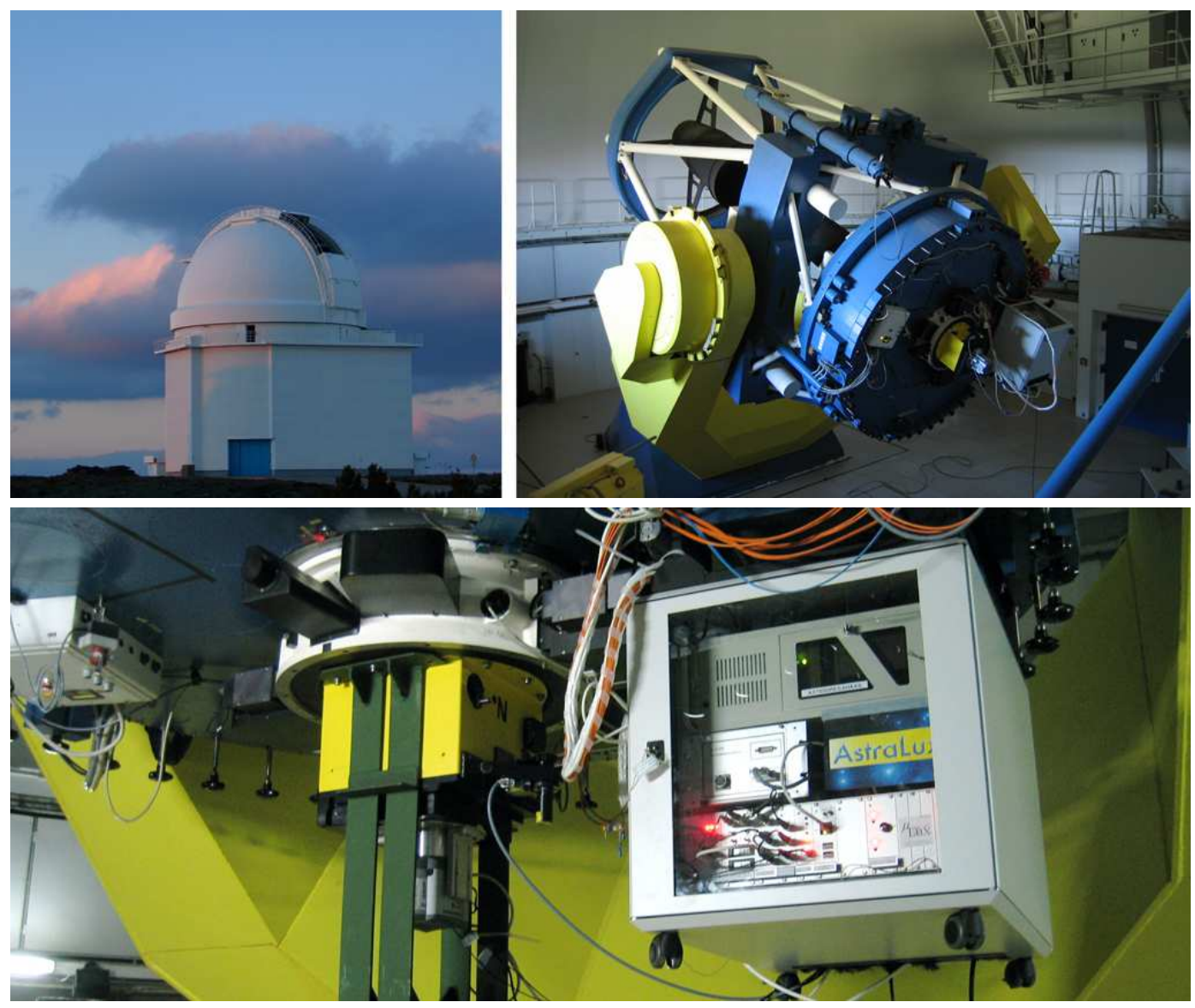

Figure 1. AstraLux and the Calar Alto 2.2-m telescope.

Top: the 2.2-m telescope dome and the telescope with AstraLux attached at the Cassegrain focus.

Bottom: Detailed view of the instrument at the telescope. The yellow box contains the 8-position filterwheel, the camera is attached at the bottom. The grey rack to the right houses the camera control computer, a keyboard/monitor combination, the filterwheel electronics, and MicroLux, the GPS timing add-on.

AstraLux routinely reaches Strehl ratios of $15-20 \%$ in the SDSS $i$ ' and $z^{\prime}$ passbands and has become the standard tool for double star observations at Calar Alto. Small technical overheads, an easy to use software interface and the availability of pipeline results in near real-time allow the observation of up to 20 targets per hour.

In the following we describe the instrument design and data processing pipeline and summarise the instrument's performance and key characteristics. Interested readers looking for more comprehensive information are referred to the diploma thesis ${ }^{13}$, which covers AstraLux and its performance in full detail.

\section{INSTRUMENT DESIGN}

\subsection{Telescope and site characteristics}

The Calar Alto observatory is located at a height of $2150 \mathrm{~m}$ in the Sierra de los Filabres, approximately $50 \mathrm{~km}$ north of Almeria in Andalusia, southern Spain. The median seeing, measured by the site's seeing monitor $\approx 300 \mathrm{~m}$ away from the telescope is 0.9 , slightly better in summer than in winter. ${ }^{14}$

Together with the given telescope diameter of $2.2 \mathrm{~m}$, the seeing defines the optimal wavelength for Lucky Imaging: the probability for the occurence of a good frame (i.e. with a Strehl ratio $>37 \%$ ) in a series of short

\footnotetext{
${ }^{\dagger}$ Available online at http://www.mpia.de/ASTRALUX/
} 
exposure times images decreases exponentially ${ }^{15}$ with the ratio of telescope diameter over Fried parameter $r_{0}$, which again depends inversely on the seeing and scales with $\lambda^{5 / 6}$. While observations at short wavelengths result in too low probabilities for usable frames, the gain in resolution by selecting only high-quality frames becomes small at long wavelengths due to the decreasing theoretical resolution of the telescope. For the Calar Alto 2.2-m telescope, the optimal wavelength for efficient Lucky Imaging lies in the range of $800-1000 \mathrm{~nm}$, including the Johnson $I$ and the Sloan Digital Sky Survey (SDSS) $i^{\prime}$ and $z^{\prime}$ passbands. At typical seeing conditions one can expect that $\approx 0.5-1 \%$ of all images will have an acceptable Strehl ratio of few $10 \%$ while providing a 6 to 10 times better resolution. A Lucky Imaging instrument for the $3.5-\mathrm{m}$ telescope at the same site would have to operate optimally in the $H$-band - a wavelength range for which no photon noise limited detectors are available off-the-shelf so far.

\subsection{Camera \& Optics}

\subsubsection{Camera Head}

The AstraLux camera head is an Andor DV887-UVB model from Andor Technologies, Belfast, Northern Ireland. It is an electron-multiplying, thinned, and back-illuminated $512 \times 512$ pixel CCD that comes as complete package with a multi-stage Peltier cooler, mechanical shutter, computer interface and software. It can be operated with readout clocks of up to $10 \mathrm{MHz}$ in frame transfer mode, allowing a full frame rate of $34 \mathrm{~Hz}$. Using subarrays, binning, and short vertical shift times allows frame rates of more than $1 \mathrm{kHz}$. These short exposure times are realised by electronic shuttering, whereas the mechanical shutter is only used for bias and dark frame acquisition and to protect the CCD window against dust. Starting at room temperature, the typical operating temperature of $-75^{\circ} \mathrm{C}$ is usually reached within 10 minutes. The camera requires neither refill of liquid coolants nor any action to maintain the vacuum inside the CCD head. The quantum efficiency (QE) of the E2V CCD97 detector peaks at $>90 \%$ in the $R$ band. At $912 \mathrm{~nm}$, the central wavelength of the SDSS $z^{\prime}$ filter, the QE is still $\approx 40 \%$.

The camera can be used either as conventional CCD without electron multiplication, or as EMCCD with multiplication gains of up to 2500 at $-75^{\circ} \mathrm{C}$. The electron gain is set from within the control software with a resolution of 255 steps, unfortunately not linearly related to the actual physical electron gainf. For Lucky Imaging we use a readout clock of $10 \mathrm{MHz}$ with $14 \mathrm{Bit} \mathrm{A} / \mathrm{D}$ resolution. The readout noise is $\approx 80 \mathrm{e}^{-}$. At an electron gain of 2500 , this corresponds to a SNR of $>30$ for single photons. So called clock-induced charges (CIC), spurious events caused during vertical clocking in the sensor or frame transfer area, occur with a probability of $\approx 5 \%$ at a vertical shift time of $1.8 \mu \mathrm{s}$. The CIC probability strongly depends on the shift time and the applied shift voltage. Both parameters can be varied via the control software, but it is not recommended to deviate from the adopted standard settings. Not only the CIC probability, but also the charge transfer efficiency depends on these parameters. At full electron gain, the camera is linear to up to 70 photons per pixel, or 170000 with disabled electron gain.

\subsubsection{Barlow Lens}

At the Calar Alto $2.2 \mathrm{~m}$ telescope, the camera's physical pixel size of $16 \mu \mathrm{m}$ roughly corresponds to almost twice the size of the theoretical PSF at $912 \mathrm{~nm}$ (SDSS $z^{\prime}$ band). Diffraction limited imaging is thus not possible without some kind of magnification optics that increases the effective focal length. Proper sampling in the sense of the Nyquist criterion can only be reached with a magnification factor of 5 in SDSS $z^{\prime}$, and even larger factors are necessary for observations at shorter wavelengths. However, for the final design, a value of 4 was adopted as a good compromise between spatial sampling and the size of the field of view which is $24 \times 24^{\prime \prime}$ at a pixel scale of $47 \mathrm{mas} / \mathrm{px}$. The hardware realisation chosen for the AstraLux instrument is a single negative achromat in Barlow configuration, i.e. placed in the optical path before the nominal focal plane of the telescope.

The AstraLux Barlow lens is a Thorlabs ACN127-030-B achromat with a diameter of $d=12.7 \mathrm{~mm}$ and a focal length of $f=-30 \mathrm{~mm}$. It is optimised and coated for the wavelength range $650-1050 \mathrm{~nm}$. The lens provides diffraction limited image quality over the full field of view and the whole specified wavelength range. Field distortion of up to $0.5 \%$ is the result of using a lens with such a short focal length in a non-telecentric configuration. Though this poses an instrumental limit to the astrometric accuracy if it is not properly calibrated, it allows a

\footnotetext{
$\ddagger$ Newer models of Andor EMCCDs allow a direct, calibrated setting of the EM gain.
} 
very compact and rigid construction of the fore-optics.

The lens is held in a standard C-mount tube - bought "off-the-shelf" like the lens itself - that is mounted directly on the camera. This guarantees a high stability of the pixel scale, which strongly depends on the distance between lens and CCD. Tolerances for tilt and de-centre of the lens were found to be $2^{\circ}$ and $1 \mathrm{~mm}$, respectively, referring to a geometric spot pattern size smaller than the diffraction limited PSF. The direct connection between camera and lens tube helps to achieve a good alignment, and eases dis- and reassembly of the camera system.

\subsubsection{Filters}

For operations at the Calar Alto $2.2 \mathrm{~m}$ telescope it was decided to refurbish the so-called Instrumenansatz 1 (IA1), an adapter that was used for mounting conventional CCDs in the past. This device can be mounted behind the video guider unit at the Cassegrain focus of the telescope and includes a filter wheel with 8 positions. This wheel can hold virtually any filter that is available at the observatorys, allowing observations at a wide range of wavelengths. The filter wheel control electronics is located in the electronics rack at the mirror cell, and the wheel is controlled via a graphical user interface on the camera control computer.

The standard filters used for Lucky Imaging observations are SDSS $i^{\prime}$ and $z^{\prime}$ band interference filters, manufactured by Asahi Spectra Co., Ltd., Tokyo, Japan. The filter transmission curves are very close to the original SDSS filter system definition ${ }^{16}$ and peak at more than $95 \%$ transmission.

\subsection{Instrument Control}

AstraLux can be controlled from virtually any point of the observatory with a $100 \mathrm{MBit}$ Ethernet network connection. The user connects via Remote Desktop Protocol (RDP) to a standard PC workstation that serves as gateway to the camera control computer. This control computer, also a standard PC, is located in the electronics rack at the mirror cell and exclusively used to run the original camera control software supplied by the vendor and a simple user interface for filter wheel control.

The camera control software offers a real time display and allows setting of all crucial parameters like exposure time, electron gain, or number of requested frames. The raw FITS data cubes (typically $18 \mathrm{MB} / \mathrm{s}$ at maximum frame rate) are not stored on the camera computer itself, but on a fast RAID-0 array with 1 TB capacity in the gateway machine.

\subsection{Data Processing \& Storage}

The AstraLux data reduction software is running on a dedicated pipeline computer, equipped with two dualcore Woodcrest processors and $8 \mathrm{~GB}$ of memory. Upon completion of an observation, the pipeline machine automatically fetches the raw data from the primary storage RAID array and starts data processing. Telescope and environmental parameters are retrieved from the Calar Alto database. The pipeline then automatically produces quicklook results of the Lucky Imaging observations in approximately the same time that is needed for data acquisition. The basic pipeline algorithms are similar to that adopted by the LuckyCam team. ${ }^{17,18}$

At the end of the observing night, complete sets of raw data exist on the gateway machine as well as on the pipeline computer. Backup is started in the morning, preferrably to an external USB harddisk, to be supplied by the observer. Up to $400 \mathrm{~GB}$ of raw data are produced in a typical winter night, while the pipeline results need only $\approx 2 \mathrm{~GB}$ of disk space.

\subsubsection{Calibration Data Processing}

Both bias and flatfield cubes are combined to master calibration images using a kappa-sigma clipping algorithm. In the case of flatfield images acquired through the conventional amplifier without electron multiplication, this will prevent cosmics from appearing in the final product. Since bias frames are usually acquired with the same camera parameters as the corresponding science observation, i.e. at high electron gains, they will most likely be contaminated by clock induced charges (CICs). With typically 50-100 single frames in a bias cube, these background events are removed by kappa-sigma clipping as well. All master calibration files are part of the final set of pipeline products.

\footnotetext{
${ }^{\S}$ See http://www.caha.es//CAHA/Instruments/filterlist.html for a list of all available filters.
} 


\section{WDS $14139+2906$}

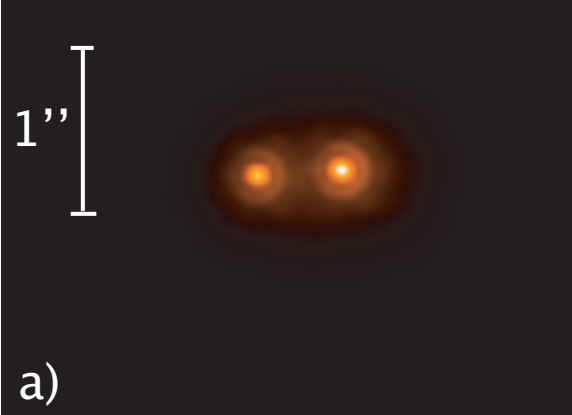

\section{WDS $15420+0027$}

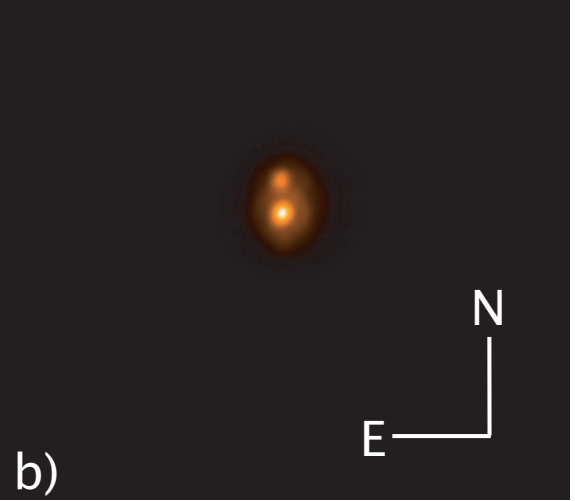

Figure 2. AstraLux first light double stars: the binary WDS $14139+2906$ with an angular separation of $0 . .52$ and component magnitudes $V=7.5$ and $V=7.6 \mathrm{mag}$, and the $0^{\prime \prime} .23$ separated binary WDS $15420+0027$ with $V=8.2$ and $V=8.8$ mag component brightnesses. Both images are based on a $2 \%$-selection from 10000 single frames in the SDSS $z$ ' filter with $30 \mathrm{~ms}$ exposure time each. Image scaling is linear.
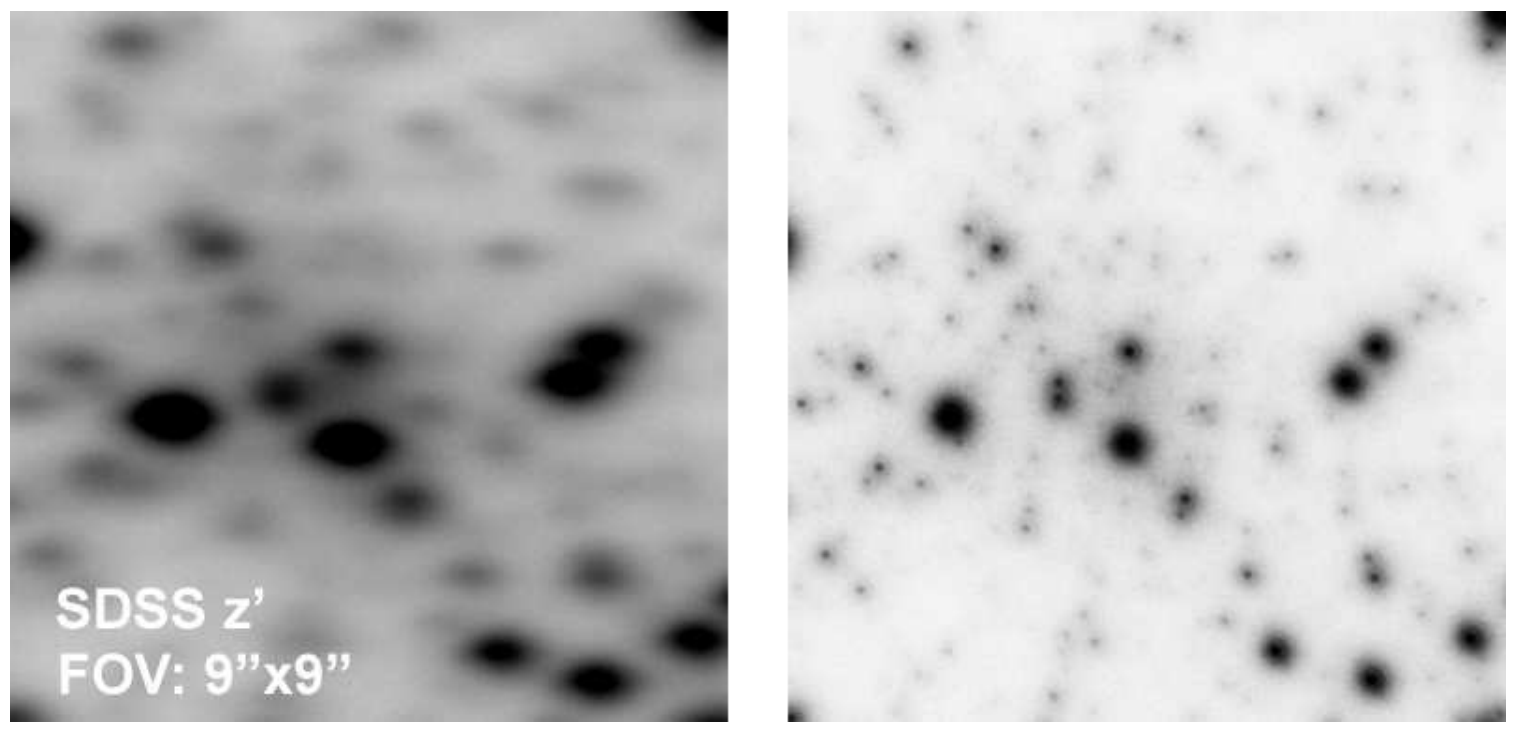

Figure 3. Comparison between seeing limited imaging and the "Lucky" version: The combination of the best $5 \%$ of 10000 single frames provided a Strehl ratio of $20 \%$ in this observation of the core of the globular cluster M15. Though the conventional result contains 20 times more photons, it is clearly inferior in terms of point source detection limits.

\subsubsection{Lucky Imaging Data Processing}

First, the position of a suitable reference object for quality assessment has to be determined. This is performed on a stacked image of the first 2 seconds of raw data, and can be done either manually or automatically. While the manual option allows to select any star just by clicking on the image, the automatic reference finding algorithm will always choose the brightest object in the field of view. The pipeline tracks the reference on all following raw data frames to account for large atmospheric tip/tilt or telescope tracking errors. 

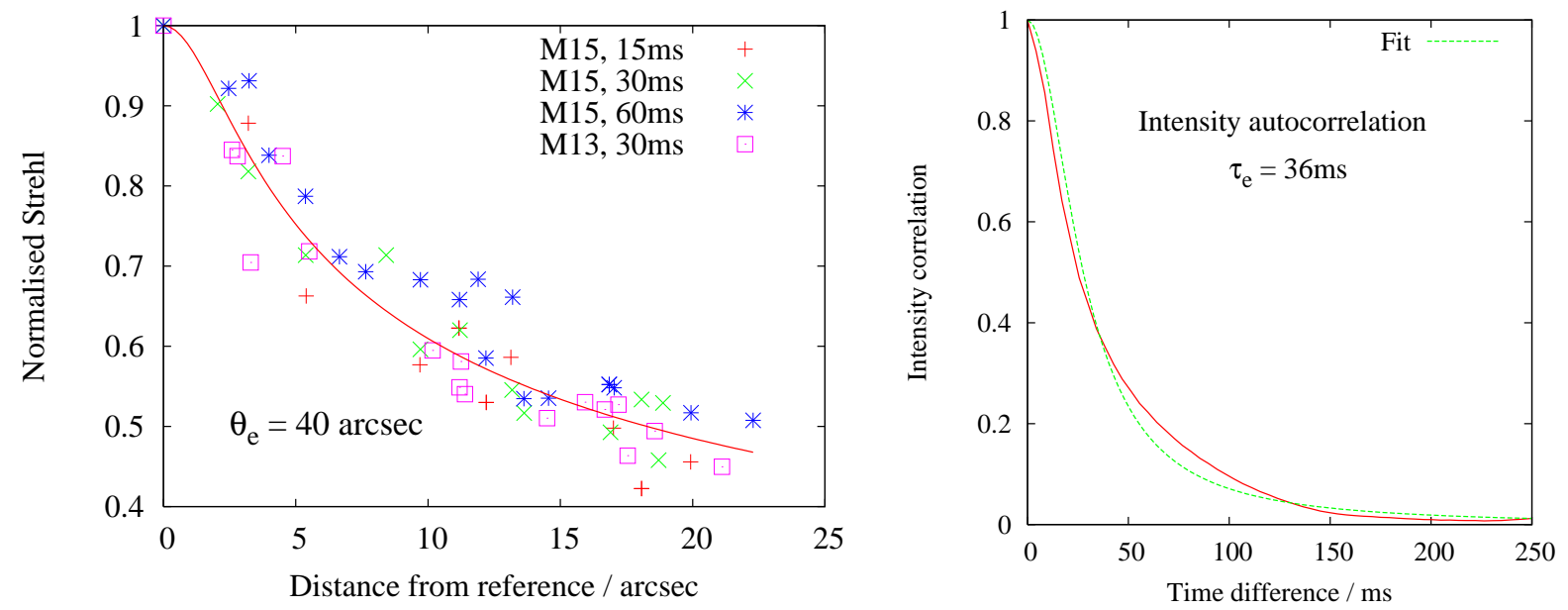

Figure 4. Left: Normalised Strehl ratio versus angular separation from the reference star for a range of single frame exposure times. All values are derived from globular cluster centre observations in the SDSS $z^{\prime}$ filter with $1 \%$ image selection rate. The given isoplanatic angle of $\theta_{e}=40^{\prime \prime}$ was derived by fitting a Moffat profile (solid line).

Right: Normalised temporal autocorrelation function of the focal plane intensity. The data was fitted with a Lorentzian profile, resulting in an estimated speckle coherence time of $\tau_{e}=36 \mathrm{~ms}$.

Subsequently, the quality of each single frame is determined by measuring the Strehl ratio of a reference object. A small region with a size of typically $32 \times 32$ pixel around the reference source is extracted, resampled by a factor of four, and noise-filtered. The resampling serves two purposes. Firstly, since the position of the brightest pixel of the reference object's PSF will be used as input to the image reconstruction algorithm, subpixel shifting can only work if the reference position is determined on resampled images. Secondly, the resampled images allow better estimates of the Strehl ratio. The Strehl value is derived from measurements of the ratio between peak flux and total flux of the reference object - a simple and fast method. Unfortunately, the peak flux in the slightly undersampled AstraLux images depends on the precise position of the PSF peak within the brightest pixel, while the total flux is independent of the reference object's position. Simulations with the AstraLux pixel scale of $\approx 47$ mas showed that Strehl measurements of a perfect PSF would suffer from a jitter of up to $20 \%$. This jitter can be reduced to $1 \%$ and less by resampling the data before measuring the Strehl ratio. If the data is not only resampled, but also filtered with the telescope's modulation transfer function (MTF), the resampling will not introduce additional noise and single-pixel events like CICs and dark current electrons can be suppressed.

The pipeline's image reconstruction module performs data reduction in its literal sense. From typically several GB of input data, just a few MB of pipeline results are produced. The most interesting ones - the Lucky Imaging results - are currently generated with the Drizzle algorithm. ${ }^{19}$ This linear reconstruction method is flux preserving and able to at least partially overcome the slight undersampling that is present in the raw data. It is capable of handling sub-pixel translations without the need to perform image shifting in the Fourier domain. The current IDL implementation of the Drizzle algorithm is somewhat simplified and does not consider image rotation or field distortions, but just shifts the selected images such that the brightest pixel of the reference star is always positioned at the same pixel coordinates. The drizzling process oversamples the input data twice, resulting in a pixel scale of $\approx 23.7 \mathrm{mas} / \mathrm{px}$ in the final images. Currently the pipeline produces drizzled results of the best 1, 2.5, 5 , and $10 \%$ of the input frames. Bias and flatfield calibration frames are applied to the input images prior to drizzling. A seeing (and tracking) limited image is generated as well using all frames to allow quick measurements of the seeing conditions, useful at times when the observatory's seeing monitor is switched off, or for later assessment of the data quality. 

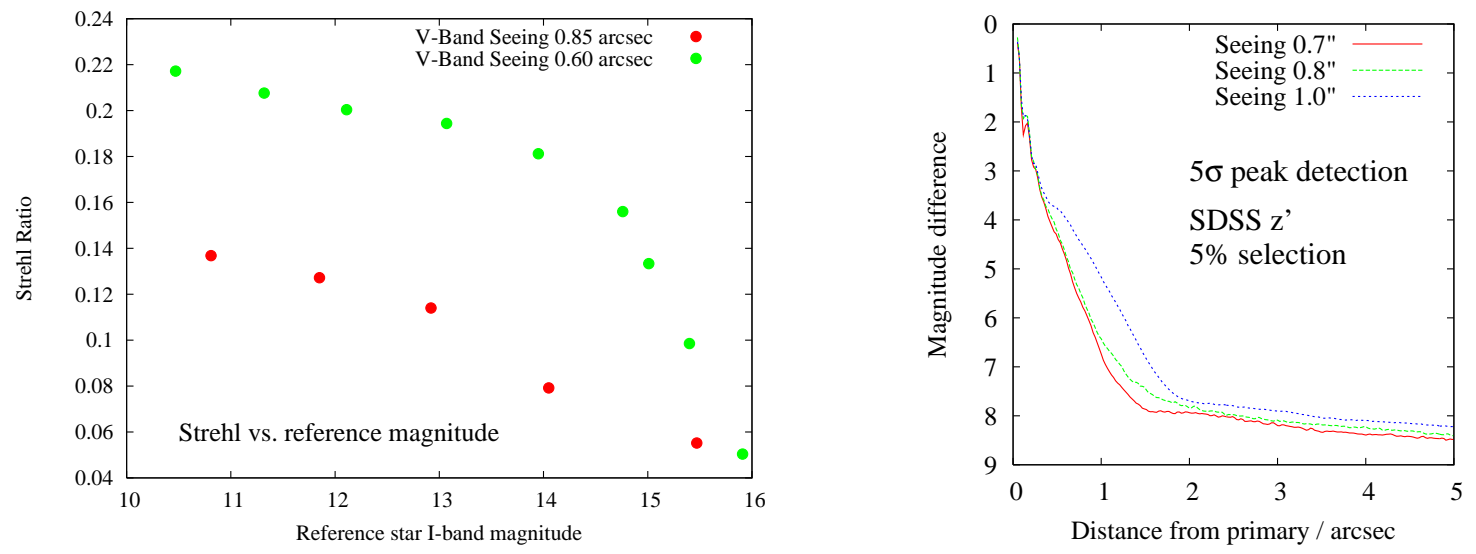

Figure 5. Left: Dependency of the final Strehl ratio on natural seeing and reference star magnitude. The plot is based on observations with $30 \mathrm{~ms}$ single frame exposure time in the SDSS $z^{\prime}$ filter and 1\% image selection rate. All Strehl ratios were measured on stars with less than $2^{\prime \prime}$ separation from the reference star.

Right: Achievable magnitude differences for a $5 \sigma$ peak detection of a fainter companion to the reference star. All curves refer to a $5 \%$ selection rate from 10000 images with $30 \mathrm{~ms}$ single frame exposure time, equivalent to an effective integration time of $15 \mathrm{~s}$. The $I$-band magnitude of all three reference stars was $\approx 10$ mag.

\section{SYSTEM PERFORMANCE}

First light at the Calar Alto 2.2-m telescope was obtained on July 6, 2006. From a total of 5 nights during the first observing run, only half a night was lost due to bad weather, and the remaining time provided photometric sky conditions with $V$-band seeing values as low as 0. . 6 .

Known bright double stars were chosen as first light targets and observed in the SDSS $z^{\prime}$ band with resulting Strehl ratios of $\approx 20 \%$ (see Figure2). Operating the instrument, and especially acquiring targets, proved to be much easier than anticipated. Though the pointing accuracy of the telescope is in general not better than $10^{\prime \prime}$, the availability of the camera's real time display allows short acquisition times of typically 1-2 min per target.

AstraLux observations of globular cluster centres enabled the characterisation of the image quality over the full field of view. Choosing different stars with a wide range of magnitudes as the Lucky Imaging reference allowed to estimate brightness limits for the reference selection and to measure the dependency of the Strehl ratio on the reference magnitude. Among the globular clusters M3, M13, and M15, the latter has been observed most extensively with AstraLux. Figure 3 shows a comparison of a Lucky Imaging result to the corresponding seeing limited image.

Astronomical observations with AstraLux started right during the very first night at the telescope, leading to two refereed publications so far. ${ }^{20,21}$ Completed and ongoing observing programs cover a wide range of target types, e.g. young stars in nearby moving groups, low-mass binaries, T Tauri binaries, transition disk objects, minor planets, and microquasars.

\subsection{Isoplanatic Angle and Coherence Time}

In the following, the isoplanatic angle $\theta_{\mathrm{e}}$ is defined as the angular separation from the reference star, where the observed Strehl ratio drops to the fraction 1/e of the reference star's Strehl ratio. The observations of centres of the globular clusters M13 and M15 allowed measurements of the Strehl ratio for a large number of stars, well distributed over the field of view. Figure 4 shows the normalised Strehl ratios, i.e. the measured Strehl ratios divided by the reference Strehl, for three different M15 and one M13 observation. The images used for this plot are based on a $1 \%$ selection from 10000 frames in the SDSS $z^{\prime}$ band. M15 was observed with three different single frame exposure times, without a significant impact on the normalised Strehl ratios. The absolute Strehl ratio slightly decreases with increasing exposure time, though, dropping from $\approx 22 \%$ at $15 \mathrm{~ms}$ to $18 \%$ at $60 \mathrm{~ms}$. 
A Moffat profile was fitted to the data, allowing extrapolation to the $1 / e$ point, and hence an estimate of the isoplanatic angle $\theta_{\mathrm{e}}$. For the SDSS $z^{\prime}$ band observations, $\theta_{\mathrm{e}}$ has been found to be $\approx 40^{\prime \prime}$, larger than the diagonal field of view size of $34^{\prime \prime}$.

This measurement has been repeated for an observation of M15 in the Johnson $I$ band. No significant difference to the isoplanatic angle extrapolated from the $z^{\prime}$ band data was found. Theory predicts a decrease of $\theta_{\mathrm{e}}$ by a factor 1.05 when switching from $z^{\prime}$ to $I$-band, too small to be reliably detected in the available data. The expected isoplanatic angle in $V$-band is $\approx 2-5^{\prime \prime}$ equivalent to $\approx 4-10^{\prime \prime}$ in the $z^{\prime}$-band, but the isoplanatic angle in speckle observations is typically about 1.7 times larger. ${ }^{22}$ The AstraLux data and comparable measurements at the $\mathrm{NOT}^{17}$ suggest that the selection process of the Lucky Imaging technique can further increase $\theta_{\mathrm{e}}$ to values as large as $40-50^{\prime \prime}$.

To determine the speckle pattern coherence time, a series of 10000 images of the bright star $\beta$ And was recorded with a time resolution of $4.6 \mathrm{~ms}$. The normalised temporal autocorrelation function is shown in Figure4 (right side). The measured coherence time $\tau_{e}=36 \mathrm{~ms}$ corresponds to the $1 / e$ point of a Lorentzian fit.

\subsection{Limiting Magnitudes}

The M13 and M15 observations were re-analysed with different choices of the Lucky Imaging reference star to assess the impact of the reference magnitude on the final Strehl ratio. Figure 5 shows the results for measurements under two different seeing conditions. While reference stars as faint as $I=15.5 \mathrm{mag}$ still allow a substantial improvement of image quality under a 0 "' 65 seeing, the same performance cannot be reached with stars fainter than 13.5 mag in 0 "' 85 seeing.

Another important number, especially in the context of binarity surveys, is the achievable contrast ratio or magnitude difference for close companions to bright host stars. This parameter was determined on final pipeline results of SDSS $z^{\prime}$ band observations under different seeing conditions. All observed stars had an $I$-band magnitude of $\approx 10 \mathrm{mag}$. The achievable magnitude differences for a $5 \sigma$ peak detection are based on measurements of the noise in concentric rings around these stars. This method suffers from the low number of available pixels in the innermost 100 mas, but is quite robust at larger angular separations. Simulations with observed PSFs were carried out to check the reliability of the numerical results.

Figure 5 shows typical detection limit plots for three different $V$-band seeing values. At angular separations larger than $2^{\prime \prime}$, the detection limit is determined by readout noise and the Poisson noise of the sky background. Using more input images, i.e. increasing the effective exposure time of the Lucky Imaging result, will increase the maximum achievable magnitude difference at large separations.
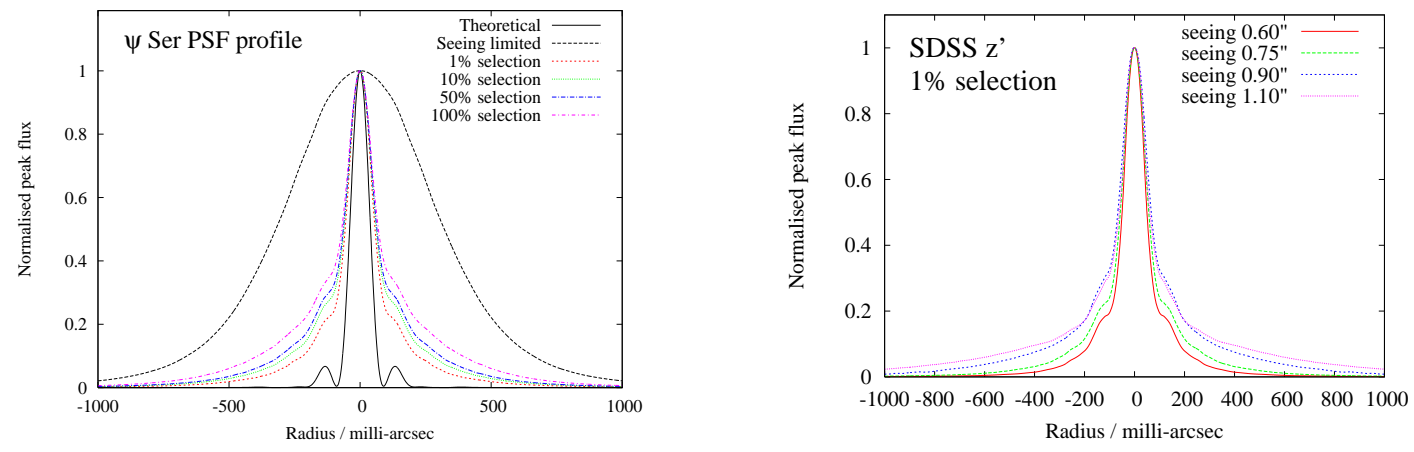

Figure 6. Left: Radial profiles of the stellar PSF for different image selection rates under 0.'75 V-band seeing. The seeing limited and diffraction limited PSF are plotted for comparison. All profiles have been normalised to a peak flux of 1. The resulting Strehl ratios for $1,10,50$, and $100 \%$ selection are $14.2,10.4,7.7$, and $6.2 \%$. The seeing limited image has a Strehl ratio of $1.9 \%$. The FWHM of the Lucky Imaging PSF core ranges from 114 to 138 mas.

Right: Radial PSF profiles for different seeing conditions. All profiles refer to $1 \%$ selection from $\geq 10000$ images with $15 \mathrm{~ms}$ single frame exposure time. Strehl ratios for V-band seeing values of $0 . \prime 60,0$.' 75,0 .' 90 , and 1.' 10 are $22.4,14.2,7.1$, and $5.2 \%$. The FWHM of the PSF core ranges from 98 to 122 mas. 

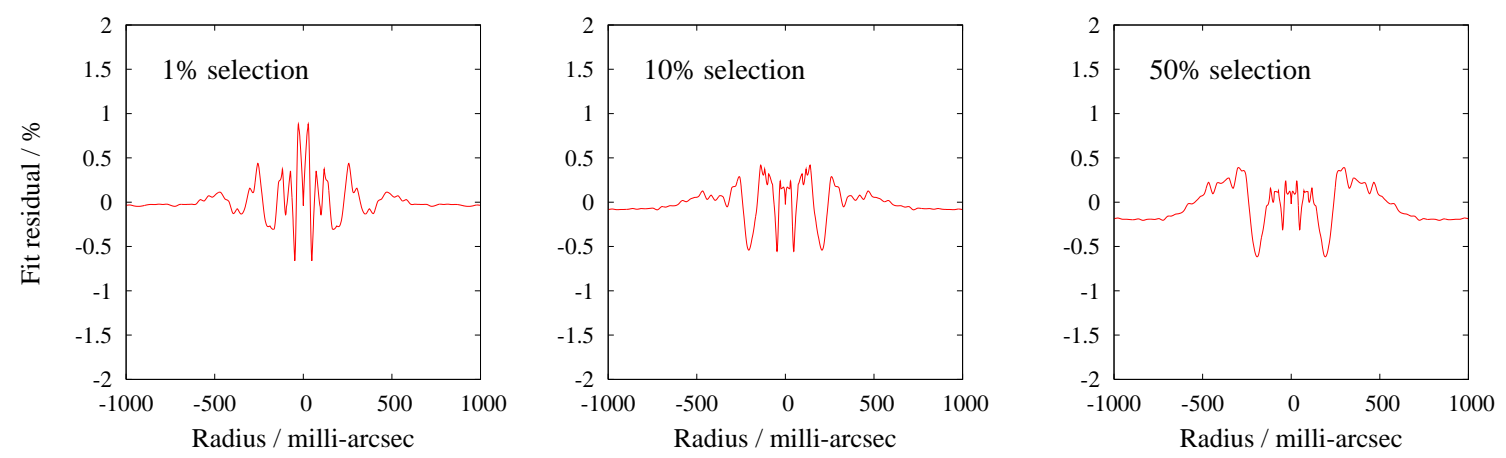

Figure 7. Fit residuals for the application of the model described by Equation 1 to the radial profiles of the $\psi$ Ser observations. The residuals are given in percent of the peak flux value.

\subsection{Point Spread Function Characteristics}

In the absence of atmospheric dispersion effects, the Lucky Imaging point spread function (PSF) of the AstraLux instrument shows a remarkable radial symmetry . There are no long-lived speckles like they are frequently observed in adaptive optics images. Using a sufficiently large number of input images - typically several thousand - efficiently averages out asymmetries. For the quantitative assessment of the PSF shape, it is therefore sufficient to consider the radially averaged profile only.

The left side of Figure 6 shows the radial PSF profile of $\psi S e r$, observed under a $V$-band seeing of 0.75 with $15 \mathrm{~ms}$ single frame exposure time through the SDSS $z^{\prime}$ filter. The profile is plotted for a range of selection rates from the 10000 input images. The theoretical and seeing limited PSF profiles are overlayed for comparison. The profile of the theoretical PSF was derived from a simulated diffraction limited image with 2.237 mas $/$ px pixel scale, whereas the raw observational data was sampled with $\approx 46.6 \mathrm{mas} / \mathrm{px}$. Though the Drizzle process of the pipeline resulted in a final pixel scale of $23.3 \mathrm{mas} / \mathrm{px}$, principally providing proper sampling in the sense of Nyquist, this is not sufficient to reconstruct a perfect PSF even in the absence of any aberrations due to atmospheric turbulence or telescope imperfections. However, for the judgement of image quality and the measurement of Strehl ratios, only the perfectly sampled PSF will be considered throughout this chapter.

To calculate the Strehl ratios, all radial profiles were interpolated on a common two-dimensional radius grid and numerically integrated to derive the total flux. The Pseudo-Strehl, i.e. the ratio of peak flux over total flux, was calculated for each profile, and divided by the Pseudo-Strehl of the theoretical PSF to obtain the real Strehl ratio. Since the radial profile of a source can be reliably reconstructed in the presence of a nearby companion or in a crowded field, this method results in more robust estimates of the total source flux - and hence Strehl ratio - than standard aperture photometry.

An increase of the selection rate causes only moderate broadening of the PSF core's FWHM, but a stronger decrease of the resulting Strehl ratio due to more pronounced PSF wings. Even at only $1 \%$ selection rate, the FWHM is considerably larger than theoretically expected. This is probably a result of the slight undersampling of the raw data. Simulations have shown that with the current setup and pipeline algorithms, the best FWHM to be expected is $\approx 95$ mas.

In a next step, the radial profiles of single stars were extracted from observational data over a range of seeing conditions. While a Strehl ratio of more than $15 \%$ can be reached under a $V$-band seeing of 0.75 and better, it rapidly drops to a few percent if the seeing gets worse than $1^{\prime \prime}$. The radial profiles for a selection rate of $1 \%$ in the SDSS $z^{\prime}$ band and a single frame integration time of $15 \mathrm{~ms}$ are plotted in Figure 6 for four different seeing values. As in the case of larger selection rates, an increase of the natural seeing leads in the first instance to more pronounced PSF wings and has only moderate effects on the FWHM of the PSF core.

"Only in one observing run in 2007 we observed static coma-like aberrations, probably related to a position-dependent tilt of the primary mirror. 


\subsection{PSF Modelling}

The additional broadening of the Lucky Imaging PSF core and the wings of the radial profiles can be modelled quite accurately as the weighted sum of a broad Moffat profile and a theoretical PSF that was convolved with a Gaussian:

$$
\operatorname{PSF}_{\mathrm{obs}}(r)=W\left(\frac{1}{r^{2} / \sigma_{\mathrm{m}}^{2}+1}\right)^{\beta}+(1-W)\left(\operatorname{PSF}_{\mathrm{th}}(r) * \exp \left(-\frac{r^{2}}{2 \sigma_{\mathrm{g}}^{2}}\right)\right)
$$

Here, W weights the two PSF components, $\beta$ is the Moffat power law index, and $\sigma_{g}$ and $\sigma_{m}$ define the widths of the Gaussian and Moffat profile, respectively. $\mathrm{PSF}_{\mathrm{obs}}$ and $\mathrm{PSF}_{\text {th }}$ refer to the observed and theoretical radial PSF profiles. This semi-analytical model has been applied to the $\psi$ Ser data presented above. The resulting fit parameters are given in Table 1, whereas Figure 7 shows the residuals.

Table 1. Semi-analytical PSF fit parameters for the $\psi$ Ser observations.

\begin{tabular}{ccccc}
\hline \hline Selection rate & $\mathrm{W}$ & $\sigma_{\mathrm{g}}[\mathrm{mas}]$ & $\sigma_{\mathrm{m}}[\mathrm{mas}]$ & $\beta$ \\
\hline $1 \%$ & 0.25 & 23.8 & 250 & 1.62 \\
$10 \%$ & 0.31 & 24.4 & 270 & 1.61 \\
$50 \%$ & 0.36 & 24.7 & 300 & 1.63
\end{tabular}

While the weighting factor and the width of the Moffat profile vary considerably under changing selection percentages, the Moffat power law index and the width of the Gaussian convolution kernel keep nearly constant. The possibility to reconstruct the observed PSF profile from the known theoretical PSF and only two model parameters is particularly interesting for binary fitting and PSF subtraction applications. In larger fields, the dependency of the model parameters on the source position could be determined, allowing PSF based photometry in crowded fields like globular cluster centres.

Figure 7 shows that the available theoretical PSF does not reproduce the second diffraction ring correctly, leaving residuals of up to $0.5 \%$ at $\approx 230$ mas distance from the PSF centre. This is possibly related to the method used for PSF simulation. Currently, the theoretical PSFs are computed for a circular aperture with central obstruction by the secondary mirror, but do not include any contributions from the secondary spider or any optics behind the primary mirror.

The described model has been applied only in its radial symmetric form so far. Further developments could include asymmetries due to atmospheric dispersion, and it might be investigated if and how accurately the model parameters can be predicted based on the seeing-limited PSF only.

\subsection{Performance summary}

AstraLux is able to reach Strehl ratios as high as $25 \%$ at the Calar Alto 2.2-m telescope in the $z^{\prime}$ band. While this is only possible under a superior seeing of $0 . \prime 6$ or better, the median Calar Alto seeing of $0 . \prime 9$ still allows to achieve Strehl ratios of more than 10\%. The typical seeing limited Strehl ratio in SDSS $z$ ' under such conditions is $\approx 1.1 \%$. In general, Lucky Imaging provides an improvement of the Strehl ratio by a factor of 10, corresponding to an increase of the signal-to-noise ratio for point sources by a factor of $10-20$, depending on atmospheric conditions. Thus a selection of only the best $5-10 \%$ of all images does definitely not have a negative effect on the detection limit for point sources.

The requirements for the reference star magnitude are similar as for observations with adaptive optics. The performance starts to significantly decrease at $I=14 \mathrm{mag}$, but image quality improvements are still possible with stars as faint as $15-16 \mathrm{mag}$. The measured isoplanatic angle in $I$-band is with $\approx 40^{\prime \prime}$ as large as typical values in $K$-band for adaptive optics observations. Lucky Imaging performs considerably better than speckle imaging techniques. The typical magnitude limit for these methods is $V \approx 12 \mathrm{mag}$, and the isoplanatic angle in $I$-band is only half as large.

The measured close companion detection limit at an angular separation of $1^{\prime \prime}$ is on average 6 mag. This is worse compared to the performance of $\mathrm{AO}$ systems - here usually 8-10 mag are reached and even more can be 
expected from future AO systems with larger actuator numbers. But: adaptive optics at 8-m class telescopes currently provides this capability only in the $H$ and $K$-band at wavelengths $>1.5 \mu \mathrm{m}$. The achievable contrast ratio in speckle imaging observations is typically two magnitudes less than for Lucky Imaging.

Single frame exposures are limited to integration times below the speckle coherence time of typically $30-40 \mathrm{~ms}$ in SDSS $z^{\prime}$. Like the size of the isoplanatic angle, this number shows seasonal variations and depends on the natural seeing conditions.

Our measurements indicate that AstraLux is comparable to LuckyCam at the Nordic Optical Telescope in terms of limiting magnitude and isoplanatic angle. Detector, optics, electronics, and software worked as expected. The simple design of the instrument certainly contributed to a smooth and satisfactory start of observations at Calar Alto.

\section{CONCLUSIONS}

Within less than one year it was possible to design, build, and characterise a Lucky Imaging instrument for the Calar Alto 2.2-m telescope. Beyond evaluation of the observing technique and data reduction strategies, scientific observations commenced right in the first night at the telescope. As a common user instrument from 2007 on, AstraLux has become the standard tool for diffraction limited imaging at the Calar Alto observatory. It is currently mostly used for binarity surveys among stars and minor planets.

The encouraging results regarding both development speed as well as scientific output triggered the start of the project "AstraLux Sur", an almost identical copy of the Calar Alto version to be used as visitor instrument at ESO's New Technology Telescope (NTT) at La Silla, Chile. AstraLux Sur is currently on its way to Chile after a development time of less than 2 months and will have first light in mid-July 2008.

Apart from Lucky Imaging, AstraLux can be used as a high speed photometer with single photon detection capability. In combination with the custom GPS timing hardware MicroLux ${ }^{23}$ it has been successfully used for observations of the optical pulse profile of the Crab pulsar.

We are currently investigating possible improvements of the optical design, namely a telecentric magnification optics and a tunable atmospheric dispersion corrector. However, the strength of the current system is its simple design, leading to short development times, low hardware costs, and high stability.

An ambitious project for the future would be the combination of AstraLux with a low-order adaptive optics system, currently developed for the Calar Alto 2.2-m telescope. This could enable observations at shorter wavelengths or increase the resulting Strehl ratio under seeing conditions normally unfavourable for Lucky

Imaging. It has recently been proven at the 5-m Palomar telescope ${ }^{24}$ that this approach actually works and can boost the performance of the Lucky Imaging technique.

\section{ACKNOWLEDGMENTS}

We are particulary grateful to Armin Böhm, Jens Helmling, and Uli Thiele as well as all technical staff at Calar Alto and MPIA for their help in preparing and commissioning AstraLux.

\section{REFERENCES}

[1] Labeyrie, A., "Attainment of diffraction limited resolution in large telescopes by fourier analysing speckle patterns in star images," $A \& \exists A \mathbf{6}, 85$ (1970).

[2] Labeyrie, A., "Speckle interferometry and possible extensions," A $\& A S$ 15, 463 (1974).

[3] Knox, K. T. and Thompson, B. J., "New methods of processing speckle pattern star images," ApJ 182, L133 (1973).

[4] Knox, K. T. and Thompson, B. J., "Recovery of images from atmospherically degraded short-time exposure photographs," ApJ 193, 45 (1974).

[5] Lohmann, A. W., Weigelt, G., and Wirnitzer, B., "Speckle masking in astronomy - Triple correlation theory and applications," Appl. Opt. 22, 4028-4037 (1983). 
[6] Jerram, P., Pool, P. J., Bell, R., Burt, D. J., Bowring, S., Spencer, S., Hazelwood, M., Moody, I., Catlett, N., and Heyes, P. S., "The LLCCD: low-light imaging without the need for an intensifier," Proc. SPIE 4306, 178-186 (2001).

[7] Hynecek, J. and Nishiwaki, T., "Recent progress toward single photon detection using charge multiplying CCD image sensors," Proc. 16th World Multiconference on Systems and Cybernetics, (2002).

[8] Mackay, C. D., Tubbs, R. N., Bell, R., Burt, D. J., Jerram, P., and Moody, I., "Subelectron read noise at MHz pixel rates," Proc. SPIE 4306, 289-298 (2001).

[9] Baldwin, J. E., Tubbs, R. N., Cox, G. C., Mackay, C. D., Wilson, R. W., and Andersen, M. I., "Diffractionlimited $800 \mathrm{~nm}$ imaging with the $2.56 \mathrm{~m}$ Nordic Optical Telescope," A\&A 368, L1-L4 (2001).

[10] Tubbs, R. N., Baldwin, J. E., Mackay, C. D., and Cox, G. C., "Diffraction-limited CCD imaging with faint reference stars," A\&3A 387, L21-L24 (2002).

[11] Tubbs, R. N., Baldwin, J. E., and Mackay, C. D., "Diffraction-limited I band imaging with faint reference stars," Proc. SPIE 4839, 1093-1102 (2003).

[12] Law, N. M., Mackay, C. D., and Baldwin, J. E., "Lucky imaging: high angular resolution imaging in the visible from the ground," $A \& A$ 446, 739-745 (2006).

[13] Hormuth, F., "AstraLux: High Angular Resolution Astronomy with an Electron Multiplying CCD," Diploma thesis, University of Heidelberg (2007).

[14] Sánchez, S. F., Aceituno, J., Thiele, U., Pérez-Ramírez, D., and Alves, J., "The Night Sky at the Calar Alto Observatory," PASP 119, 1186-1200 (2007).

[15] Fried, D. L., "Statistics of a Geometric Representation of Wavefront Distortion," JOSA 55, 1427-1435 (1965).

[16] Fukugita, M., Ichikawa, T., Gunn, J. E., Doi, M., Shimasaku, K., and Schneider, D. P., "The Sloan Digital Sky Survey Photometric System," AJ 111, 1748 (1996).

[17] Tubbs, R. N., "Lucky Exposures: Diffraction Limited Astronomical Imaging Through the Atmosphere," $\mathrm{PhD}$ thesis, Cambridge University (2003).

[18] Law, N. M., "Lucky Imaging: Diffraction-Limited Astronomy from the Ground in the Visible," PhD thesis, Cambridge University (2006).

[19] Fruchter, A. S. and Hook, R. N., "Drizzle: A Method for the Linear Reconstruction of Undersampled Images," PASP 114, 144-152 (2002).

[20] Hormuth, F., Brandner, W., Hippler, S., Janson, M., and Henning, T., "Direct imaging of the young spectroscopic binary HD 160934," A $\& A$ 463, 707-711 (2007).

[21] Sicilia-Aguilar, A., Merín, B., Hormuth, F., Ábrahám, P., Henning, T., Kun, M., Patel, N., Juhász, A., Brandner, W., Hartmann, L. W., Csizmadia, S., and Moór, A., "The Rapid Outbursting Star GM Cep: An EXor in Tr 37?," ApJ 673, 382-399 (2008).

[22] Vernin, J. and Munoz-Tunon, C., "Optical seeing at La Palma Observatory. 2: Intensive site testing campaign at the Nordic Optical Telescope," A\&A 284, 311-318 (1994).

[23] Hormuth, F., "MicroLux: high-pecision timing of high-speed photometric observations," Proc. SPIE, this volume (2008).

[24] Law, N. M., Mackay, C. D., Dekany, R. G., Ireland, M., Lloyd, J. P., Moore, A. M., Robertson, J. G., Tuthill, P., and Woodruff, H., "Getting Lucky with Adaptive Optics: Fast AO Image Selection in the Visible with a Large Telescope," eprint arXiv:0805.1921 (2008). 